\title{
El tema de urgencia en los debates en torno al estado de la nación
}

\author{
Luis Cortés Rodríguez* \\ Universidad de Almería, España \\ Centro de Investigación "Comunicación y Sociedad" (CySOC)
}

\begin{abstract}
Resumen
Parece evidente que, a la hora de analizar intervenciones extensas (conferencias, discursos presidenciales, etc.), las unidades discursivas orales hasta ahora más estudiadas (enunciado, acto, subacto) resultan, por reducidas, poco eficaces, al menos a la hora de fijar cuestiones relacionadas con el plano secuencial. Este artículo pretende esbozar unas categorías superiores de división, así como su aplicación a los "Debates en torno al Estado de la Nación", debates políticos que se celebran anualmente en España. Para ello, examinamos la secuencia inicio, y más concretamente uno de los cuatro temas en que se divide esta: el tema de urgencia. El estudio de sus estructuras temáticas y de procesamiento, así como los mecanismos (determinados conectores continuativo-reforzadores, adjetivos, series enumerativas, etc.) empleados para manifestar ciertos propósitos en las intervenciones que forman nuestro corpus son las cuestiones de las que aquí tratamos.

Palabras clave: discurso oral, unidades de segmentación, análisis del discurso político.
\end{abstract}

*Para correspondencia, dirigirse a: Luis Cortés Rodríguez (1cortes@ual.es), Departamento de Filología, Facultad de Humanidades, Universidad de Almería, Almería 04120, España. 


\title{
The Topic of URGency IN DEBATES CONCERning THE STATE
} OF THE NATION

\begin{abstract}
The spoken discursive units most extensively studied thus far (i.e. utterance, act, sub-act) prove too simple and thus inefficient when analyzing long interventions (e.g. lectures, presidential addresses, etc) in general and issues impinging on their sequential nature. This article puts forth a number of broader-scale segmentation categories and deals with their application to the analysis of debates concerning the state of nation, a political debate that is held every year in Spain. To this end, we concentrate on the beginning sequence and, more specifically, on one of the four topics into which this is divided, namely, the topic of urgency. In particular, we pay special attention to the topic and processing patterns as well as to the devices deployed in the interventions in our corpus to achieve specific communicative goals, such as the choice of continuing-reinforcing connectors, adjectives, listing series, etc.
\end{abstract}

Key words: oral discourse, units of segmentation, sequential plane, analysis of political discourse.

Recibido: $31 / 08 / 13 \quad$ Aceptado: 08/10/13

\section{INTRODUCCIÓN ${ }^{1}$}

1.1. En un artículo reciente (Cortés 2012a) expusimos nuestra tipología de las unidades del discurso oral. Aunque el cuadro que ofrecemos a continuación muestre ligeras modificaciones ${ }^{2}$ tanto en el esquema como en algunos aspectos terminológicos con respecto al del citado artículo, las nociones y la tipología de las unidades siguen siendo las mismas. Se mantienen las diferencias entre unidades del plano secuencial (discurso

1 Este trabajo forma parte de proyecto FFI2012-31699, "Los debates en torno al estado de la nación (1983-2011). Análisis pragmalingüístico", concedido por el Ministerio de Economía y Competitividad de España.

2 Mi agradecimiento a M. ${ }^{a}$ Matilde Camacho por sus observaciones sobre esta cuestión. 
procesado) y las del plano enunciativo (discurso en procesamiento); las primeras, relacionadas con las actividades temático-textuales, son las secuencias (inicio, desarrollo y cierre) y las subsecuencias (temas, subtemas, etc.); las segundas, relacionadas con lo ilocutivo-textual, son los enunciados (actos y subactos). Cfr. cuadro $\mathrm{n}^{\mathrm{o}} 1$ :

\section{CuADro $\mathrm{n}^{\circ} 1$. Unidades del discurso oral}

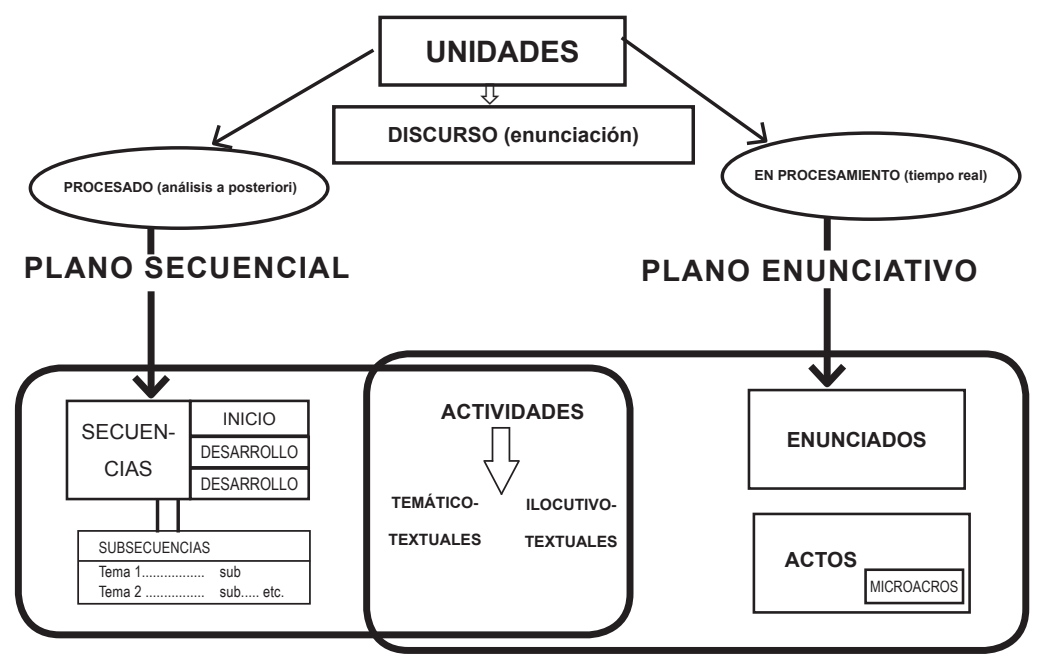

Es sabido que hasta nuestros días, la mayoría de los trabajos sobre el discurso oral en español (en otras lenguas, igualmente) se han centrado en el plano enunciativo (cortesía, marcadores del discurso, actos de habla, argumentación, orden de palabras, etc.); por el contrario, son mínimos ${ }^{3}$ aquellos otros basados en actividades temático-textuales (plano secuencial), o sea, en el análisis de un discurso en su compleción: sus secuencias, la tipología de sus temas, subsubtemas, etc.

3 En un artículo reciente sobre el género conferencia (Robles Garrote 2013), publicado en esta misma revista, se ha aludido a la escasez de análisis a partir de este plano secuencial nuestro, coincidente, en parte, con la macroestructura de la autora: "La mayor parte de los estudios que han abordado la macroestructura textual se centran en el discurso escrito y por este motivo son pocas las herramientas metodológicas específicas del discurso oral formal para analizar este aspecto en los géneros discursivos orales" (pág. 132). 
1.2. A cuestiones generales acerca de los inicios, nos hemos referido en un artículo de próxima aparición (Cortés, en prensa). En él, hacemos hincapié en la importancia de inicios y cierres en cualquier tipo de discurso, pues si con los primeros hemos de pretender ganar el interés del público y empezar a crear una buena imagen, con los cierres se perseguirá el remate de esa buena imagen mediante posibles mecanismos ornamentales, a la par que se intentará incidir sobre aquellos puntos considerados como más importantes entre los tratados. En el discurso político, especialmente en estos que analizamos, los Debates en torno al estado de la nación (en adelante DEN), se suele sacrificar la brillantez por la eficacia de los datos.

Los DEN se celebran, anualmente, en España y generan siempre un gran interés, pues Gobierno y oposición abordan los asuntos políticos más importantes del país ${ }^{4}$. En este artículo, pretendemos acercarnos a uno de los temas que forman parte del inicio en los discursos estudiados: el denominado por nosotros tema de urgencia.

Aunque no sea algo que esté establecido y que, por ende, encontremos en todas las intervenciones analizadas, hay una marcada tendencia a segmentar la secuencia inicio en cuatro temas; uno inicial, tema de urgencia, que aparece cuando algún acontecimiento reciente se convierte en importante noticia para el país (conflicto con Marruecos por el islote de Perejil, muerte de turistas españoles en Yemen, de nuestros soldados en Afganistán, etc.); un segundo, que tratará sobre la justificación del discurso; el tercero se asocia con la aparición de un tema de interés mediante el cual se da una importancia máxima a un asunto, por motivos diversos, bien por parte del Gobierno (en 2003, 2005, 2007, 2009, 2010 y 2011) bien por la oposición

\footnotetext{
4 Instaurados por el socialista Felipe González, en 1983, -en la II Legislatura- su celebración es anual, y desde entonces solamente se han dejado de llevar a cabo en seis ocasiones, todas ellas por la convocatoria de elecciones generales. Esta aproximación al estudio de los "Debates" que ofrecemos aquí, en cuanto a su extensión, se basa en un corpus delimitado en dos aspectos. En primer lugar, vamos a partir de los DEN celebrados en el presente siglo, o sea desde 2001 -en 2000 no hubo- hasta el último, el efectuado en 2011; en total 9 debates, que abarcarán tres legislaturas completas (VII, VIII y IX). En la primera de ellas, el Gobierno estaba presidido por J.M. Aznar y era Rodríguez Zapatero el líder de la oposición; se celebraron los debates en 2001, 2002 y 2003; en las dos restantes, Rodríguez Zapatero y M. Rajoy se reparten estos papeles (2005, 2006 y 2007, 2009, 2010 Y 2011). En segundo lugar, en este trabajo, la limitación viene impuesta por el contenido: nos vamos a ceñir a los discursos iniciales de los presidentes (Aznar y Rodríguez Zapatero) y de los líderes de la oposición (Zapatero y Rajoy) Para ello, nos valdremos de las transliteraciones aparecidas en el diario de sesiones, así como de los vídeos correspondientes. Por tanto, el corpus a partir del cual se ha llevado a cabo este acercamiento consta de nueve discursos presidenciales de, aproximadamente, unos sesenta minutos de duración media y otros tantos del líder de la oposición, de unos cincuenta minutos de duración media.
} 
(en todos los debates). La cuarta cuestión (tema), que cierra el inicio, se dedica a adelantar los aspectos que se van a tratar a lo largo de la secuencia siguiente, la más larga de un discurso, la secuencia desarrollo: unos políticos los denominarán ejes, en 2002 y 2006, otros, objetivos, en 2003, balance, en 2001, 2005 o 2007, etc. Nosotros, en este artículo, vamos a ocuparnos del primero de esos cuatro temas: el tema de urgencia ${ }^{5}$.

\section{TEMA DE URGENCIA}

El tema de urgencia, decíamos, viene dictado por la importancia de un hecho acontecido de forma imprevista e inmediata en el tiempo. En este siglo, dos tipos diferentes de motivos han sido considerados en los DEN: $a$ ) algún accidente mortal, bien civil (como el ocurrido en Ferrol, La Coruña [Rajoy, 2005]) bien resultado de actos terroristas, a turistas en Yemen [Zapatero, 2007 y Rajoy, 2007] o a militares (como el acontecido en Afganistán [Zapatero, 2011 y Rajoy, 2011]), y b) algún grave acontecimiento político; uno de ellos, el ya citado del islote de Perejil (con el consiguiente problema en las relaciones con Marruecos) fue tratado por el Gobierno y la oposición [Aznar, 2002; Zapatero, 2002], en tanto que el otro, el tema vasco, lo fue en dos debates distintos, ambos por parte de la oposición; en un caso, para apoyar una decisión de un Gobierno conservador [Rodríguez Zapatero, 2002]; en el otro, con la derecha en la oposición [Rajoy, 2006], para mostrar su desconfianza y desacuerdo con la actitud del Gobierno socialista ante ETA. En tres ocasiones el presidente y en cinco el líder de la oposición iniciaron sus discursos con temas de urgencia. Cfr. cuadro $\mathrm{n}^{\circ} 2$ :

Cuadro $\mathrm{n}^{\circ}$ 2. Temas de urgencia en los DEN del presente siglo

\begin{tabular}{|ccccc|}
\hline \multicolumn{5}{c|}{ TEMAS DE URGENCIA } \\
\hline AÑos & Presidente & TeMA & $\begin{array}{c}\text { Líder DE LA } \\
\text { Oposición }\end{array}$ & Tema \\
\hline 2002 & Aznar & Perejil & Zapatero & $\begin{array}{c}\text { Perejil Parlamento } \\
\text { vasco }\end{array}$ \\
\hline
\end{tabular}

5 En Cortés (en prensa) puede verse el cuadro general con las apariciones de cada uno de los cuatros temas en el presidente del Gobierno y en el líder de la oposición. 


\begin{tabular}{c|c|c|c|c}
\hline 2005 & \multicolumn{2}{|c}{ Rajoy } & Ferrol \\
\hline 2006 & & & Rajoy & $\begin{array}{c}\text { Negociación } \\
\text { con ETA }\end{array}$ \\
\hline 2007 & Zapatero & Yemen & Rajoy & Yemen \\
\hline 2011 & Zapatero & Afganistán & Rajoy & Afganistán \\
\hline
\end{tabular}

Estas intervenciones de urgencia no tienen un esquema determinado, ni siquiera un tipo de discurso que mantenga o repita ciertas formas o mecanismos; al contrario, son fragmentos heterogéneos tanto en el contenido como en la estructura. Tal heterogeneidad la podemos observar, por ejemplo, si comparamos las intervenciones del presidente y del líder de la oposición ante la muerte de turistas españoles en Yemen [2007] o de soldados en Afganistán [2011].

En 2007, con siete personas muertas (eran turistas y no militares), el presidente Zapatero se limitó casi a una mera nota informativa mediante la cual expuso, ante los diputados, el pesar de todos por las víctimas del atentado criminal $^{6}$. Su discurso, estructuralmente, nos ofreció un tema dividido en dos subtemas sin conexión alguna entre ellos; en el primero, dedicado al pésame, el líder socialista emplea estrategias discursivas muy comunes y propias de cualquier intento de manifestación de autoridad; así, en el inicio, utiliza, igual que hará el líder de la oposición, mecanismos propios de la asertividad: la primera persona, el verbo querer o el adjetivo posesivo mis:

(1) Señor presidente, señorías, quiero que mis primeras palabras sean para expresar en ...

También recurre afectivamente a otra estrategia común en el discurso político: la inclusión, al utilizar muestras de solidaridad compartida:

(2) $[\ldots]$ que mis primeras palabras sean para expresar en nombre del Gobierno -sé también que de toda la Cámara y de la sociedad española- nuestro

6 Señor presidente, señorías, quiero que mis primeras palabras sean para expresar en nombre del Gobierno -sé también que de toda la Cámara y de la sociedad española- nuestro pesar por el asesinato de siete españoles en Yemen víctimas de un atentado criminal y el deseo, junto con la condolencia a las familias, de la recuperación de los otros compatriotas que han resultado heridos. Como saben, a estas horas viajan el ministro de Industria, Turismo y Comercio y el secretario de Estado de Política Exterior, acompañados por equipos médicos y de la Policía científica, para facilitar y dirigir las tareas de repatriación de nuestros compatriotas. 
pesar por el asesinato de siete españoles en Yemen víctimas de un atentado criminal

Tal escasez de afectividad hace que su tema de urgencia resulte distante, tanto en este primer subtema como en el segundo, en el que se limita a informar de la repatriación de los fallecidos. De modo diferente, el líder de la oposición, Mariano Rajoy, manifestó una mayor emotividad, tanto en su léxico (la repetición de términos como asesinatos) o en la adjetivación (terribles atentados), como en el empleo de determinados mecanismos de énfasis (la serie enumerativa ${ }^{7}$ ), especialmente a la hora de expresar su idea principal; esta no es otra que la necesidad de perseverar en la lucha:

(3) Hoy es el momento

del duelo,

de transmitir a las familias

nuestro apoyo y

nuestra solidaridad,

de acompañarles en su dolor,

pero también es el momento de decir a los asesinos que

ni vamos a claudicar

ni vamos a admitir la intimidación

El líder del Partido Popular une a los siete turistas los cinco soldados españoles muertos, diez días antes, en el Líbano, lo que no deja de ser una manera de 'recordar' a los actuales dirigentes su negativa, cuando estaban en la oposición, a participar en conflictos bélicos:

(4) ayer otros siete españoles fueron asesinados en un atentado terrorista en Yemen. En apenas diez días, trece compatriotas -y digo compatriotas porque los tres soldados colombianos asesinados en Líbano llevaban la bandera de nuestra patria- han muerto en atentados perpetrados por terroristas yihadistas

En 2011, la muerte de dos soldados españoles en Afganistán produjo reacciones encontradas y con tratamiento distinto al de cuatro años antes. Es ahora Zapatero quien se ve en la necesidad de dar una explicación más amplia y sentida. Secuencialmente, el tema se segmenta en tres subtemas, cada uno de los cuales realiza una función en lo que ya denominamos, en trabajos anteriores ya aludidos, la disposición interna de las unidades 
secuenciales ${ }^{8}$. En el cuadro $\mathrm{n}^{\circ} 3$ podemos ver tanto dicha disposición como las unidades temático-textuales y de procesamiento en que se divide este tema de urgencia:

\section{Cuadro $n^{0}$ 3. Zapatero [2011]. Disposición interna del tema de urgencia en el inicio}

\section{PRESENTACIÓN DEL TEMA}

Subtema 1. Señor presidente, señoras y señores diputados [subacto 1], quiero mostrar el profundo pesar del Gobierno por los dos atentados que en muy pocos días han sufrido en Afganistán las Fuerzas Armadas españolas [subacto 2] [acto 1].

\section{RAZONES QUE SOSTIENEN TAL OPINIÓN}

Como consecuencia de ellos [subacto 3] han perdido la vida el sargento Manuel Argudin Perrino y la soldado Niyireth Pineda Marín [subacto 4] [acto 2]. También han resultado gravemente heridos el teniente Agustín Gras Báez y los soldados Jennifer García López, Abián Quevedo Santana, Ibrahim Maanan Ismael, Ruben Velázquez Herrera, Jhony Alirio Herrera Trejos y Roi Villa Souto, y el intérprete civil Menan Latifi Ashjari [acto 3] [ENUNCIADO 1].

Subtema 2. Que la mención de sus nombres sea muestra del homenaje a su memoria, de la solidaridad con sus familias y del recuerdo a todos los que, miembros de los Ejércitos, de la Guardia Civil, de la Policía Nacional, de la Administración y de distintas organizaciones sociales [subacto 5], contribuyen a la paz y seguridad mundial [subacto 6] [acto 4] y al auxilio de poblaciones maltratadas por la guerra, la violencia o el ansia de libertad [acto 5] [ENUNCIADO2].

8 Ya hemos analizado en trabajos anteriores cómo los temas, subtemas (incluso en alguna ocasión los susbsubtemas) tienen en muchas ocasiones su propia estructura interna, que es su división en tres partes: a) presentación del tema, b) razones que justifican, y c) constatación del hecho y consecuencias. 


\section{CONSTATACiÓN DEL HECHO O CONSECUENCIAS}

Subtema 3. Afganistán es hoy el más expresivo ejemplo de la necesidad de intervención de la comunidad internacional en escenarios convulsos [subacto 7] donde se ha perdido el valor de la vida [subacto 8] y donde se desconoce el sentido mismo de los derechos humanos [subacto 9] [acto 6]. Es también el que más esfuerzos exige [subacto 10], el que más sufrimiento causa [subacto 11], el más violento [subacto 12], el más desolador [subacto 13] [acto 7]. Pero también la comunidad internacional está conociendo experiencias esperanzadoras [acto 8], así ocurre en el Mediterráneo sur y también en el Medio Oriente [subacto 14], donde la libertad se abre caminos inesperados a los que España, como hizo desde su primera manifestación, prestará toda la ayuda que sus pueblos soliciten [subacto 15] [acto 9] [enunciado3].

\begin{tabular}{|c|c|c|}
\hline \multicolumn{3}{|c|}{ RESUMEN DE UNIDADES ILOCUTIVO-TEXTUALES Y DE PROCESAMIENTO } \\
\hline Temas & SUB(SUB)TEMAS & ENUNCIADOS Y (SUB) ACTOS \\
\hline & Subtema 1: Dolor & $\begin{array}{r}\text { Enunciado } 1 \text { [acto } 1 \\
(\operatorname{subactos} 1,2]]\end{array}$ \\
\hline $\begin{array}{l}\text { TEMA1. } \\
\text { Zapatero [2011]. }\end{array}$ & $\begin{array}{l}\text { Subtema 2: } \\
\text { Homenaje }\end{array}$ & $\begin{array}{l}\text { Enunciado } 1 \text { [ } 2 \text { (subactos } \\
\text { 3, 4) y 3] } \\
\text { Enunciado } 2 \text { [actos } 4 \\
\text { (subactos 5, 6) y } 5 \text { ] }\end{array}$ \\
\hline Afganistán & $\begin{array}{l}\text { Subtema 3: Resumen } \\
\text { actuación }\end{array}$ & $\begin{array}{l}\quad \text { Enunciado } 3 \text { [actos } 6 \\
\text { (subactos } 7,8,9) 7 \text { (subactos } \\
\text { 10, 11, 12, 13) } 8 \text { y } 9 \text { (subactos } \\
14 \text { y 15)] }\end{array}$ \\
\hline
\end{tabular}

Rajoy, extrañamente, siendo una cuestión que alude a soldados españoles muertos en acción bélica, solo emplea ochenta y seis palabras ${ }^{9}$ para un tipo de mensaje que es un ejemplo de mera nota informativa sobre su pesar y el del partido que representa.

9 Permítanme, señorías, que antes de comenzar mi intervención me sume a la tristeza que experimentan los españoles por los dos militares que acaban de morir en acto de servicio durante una acción bélica en Afganistán. Ellos y sus compañeros heridos merecen el homenaje de toda la nación, al que yo quiero aportar el de mi grupo parlamentario y el mío personal. Y desde aquí quiero enviar un cariñoso recuerdo a los familiares y compañeros de los fallecidos y nuestro deseo de pronta recuperación a los convalecientes. 
Las diferencias entre las cuatro intervenciones se dan tanto en el plano secuencial (en cuanto a temas, subtemas y disposición interna), como en el plano enunciativo, es decir, en la selección de mecanismos y formas con que conseguir los efectos discursivos perseguidos (pesar, cortesía, emoción, etc. $)^{10}$. Así, ciñéndonos, por cuestión de espacio, a los dos temas de inicio de Zapatero aquí señalados [2007 y 2011], podemos observar algunos de tales contrastes. En 2007, el presidente socialista utiliza el término pesar:

(5) Señor presidente, señorías, quiero que mis primeras palabras sean para expresar en nombre del Gobierno -sé también que de toda la Cámara y de la sociedad española-nuestro pesar por el asesinato

\section{En 2011, en cambio, será el profundo pesar:}

(6) Señor presidente, señoras y señores diputados, quiero mostrar el profundo pesar del Gobierno por los dos atentados que en muy pocos días han sufrido en Afganistán las Fuerzas Armadas españolas

10 Hemos de aclarar con Cortés y Camacho (2005: 96) que no se puede confundir el enunciado unidad de procesamiento - mezcla de textualidad e interacción-con unidad temática -solo textualidad-. Por ejemplo, la intervención-respuesta en el siguiente fragmento de Zapatero [Debate en torno al estado de la nación 2002]:

Subtema 2. Ninguna mayoría puede vulnerar las reglas del juego [acto 1] y por tanto el Partido Socialista va a ser, como siempre, firme defensor de la Constitución, del Estatuto de Autonomía del País Vasco y de las reglas del juego que en ella se contienen [acto 2]. Esa es la garantía de nuestras libertades [acto 3 ]. (Aplausos.) Y diré algo más, señorías (subacto 1]. Todos los que se sienten demócratas deberían tener un objetivo esencial: [subacto 2] poner toda la energía en la defensa de la vida, de la libertad, de la seguridad, de los concejales del Partido Popular y del Partido Socialista, de los concejales del Partido Socialista y del Partido Popular y de todos los ciudadanos que en Euskadi sufren amenaza y chantaje [subacto 3] [acto 4]. [ENUNCIADO 1] Ese es nuestro compromiso esencial [subacto 4] y debería de ser -espero que lo sea definitivamente [acto 6]- el de todos aquellos que son [subacto 5] y se sienten demócratas ante todo [subacto 6], que es el compromiso de defender la libertad de todos `subacto 7] y de los que se sienten amenazados [subacto 8] [acto 5 ]. [ENUNCIADO2],

pertenecerá a una misma unidad temática, un mismo subtema, pero esta este se manifestará mediante dos intentos comunicativos diferentes, dos unidades (enunciados) distintas que acarrean unas relaciones personales determinadas, tanto mediante actividades temáticas (continuar y desarrollar el tema) como de índole más afectiva (ser educado, saber escuchar y responder a las expectativas de A, atendiendo a sus necesidades comunicativas, etc.). Tras "chantaje" hay una larga pausa que se justifica por la sensación de totalidad conclusa que siente el emisor y que transmite al receptor; la nueva intención comunicativa solo se vincula con la anterior mediante el subtema, pero su estructura no tiene relación con el cotexto previo. En estos casos, hablaremos de enunciados diferentes pertenecientes a una misma unidad temática, en este caso una subsecuencia. 
No solamente es más larga la intervención de 2011 (261 palabras frente a las 172 de 2007) sino que, como hemos señalado más arriba, contrariamente a la simple notificación hecha en 2007, ahora, en 2011, se activan recursos más formales con los que dar al discurso una mayor solemnidad. Así, Zapatero emplea estructuras complejas con un sujeto formado por una proposición seguido de verbo en subjuntivo: que la mención de su nombre + sea como introducción a un amplio enunciado constituido por una larga serie enumerativa mediante la cual se quiere realzar la gravedad del acto, a la par que enfatizar la mención de los nombres de las víctimas y heridos -lo que no había ocurrido con los siete caídos en Yemen [2007]. Tal mención no solo sirve para reforzar el sentimiento ante la desgracia de los fallecidos en una situación bélica (aunque ya se encargue el presidente del Gobierno de aclarar que ha ocurrido en auxilio de poblaciones maltratadas), sino que también vale para realzar sus palabras posteriores, que Zapatero expresa mediante esta compleja serie enumerativa:

(7) Que la mención de sus nombres sea muestra del homenaje a su memoria, de la solidaridad con sus familias y del recuerdo a todos los que miembros de los Ejércitos, de la Guardia Civil, de la Policía Nacional, de la Administración y de distintas organizaciones sociales, contribuyen a la paz y seguridad mundial y al auxilio de poblaciones maltratadas por la guerra, la violencia o el ansia de libertad

Ya hemos analizado en otros lugares el valor tanto argumentativo como enfático de este mecanismo (Cortés 2007, 2012b), tan empleado, por ejemplo, a la hora de buscar el aplauso. Como contraposición a tanto dolor y como justificación de nuestra presencia pacificadora en el conflicto, el presidente termina su actuación con un acto que representa un argumento anti-orientado e introducido por un pero: "Pero también la comunidad internacional está conociendo experiencias esperanzadoras, así ocurre en el Mediterráneo sur y también en el Medio Oriente ...", antiorientación argumentativa que invierte la conclusión esperada, pues a pesar de las muertes, nuestras tropas han de permanecer allí. 
Bien es verdad que es en los dos temas de urgencia que tienen el País Vasco como centro de interés [Zapatero, 2002 y Rajoy, 2006] donde se perciben con mayor claridad los mecanismos encaminados a crear un discurso cuyas ideas quieren ser además de firmes, convincentes.

Zapatero, en 2002, dispone el tema de forma interna tripartita, o sea: presentación del tema, razones y constatación del hecho y consecuencias. En el cuadro $\mathrm{n}^{\circ} 4$ se puede observar tanto dicha disposición como las unidades temático-textuales y de procesamiento en que se divide el tema:

\section{Cuadro $n^{\circ} 4$. Zapatero [2002]. Disposición interna del tema de urgencia en el inicio}

\section{PRESENTACIÓN DEL TEMA}

Subtema 1. En segundo lugar, ante la decisión del Parlamento vasco, quiero reiterar con toda contundencia nuestro compromiso firme en la defensa de la Constitución y del estatuto de autonomía [ENUNCIADO 1].

\section{RAZONES QUE SOSTIENEN TAL OPINIÓN}

Subtema 2. Ninguna mayoría puede vulnerar las reglas del juego [acto 1] y por tanto el Partido Socialista va a ser, como siempre, firme defensor de la Constitución, del Estatuto de Autonomía del País Vasco y de las reglas del juego que en ella se contienen [acto 2]. Esa es la garantía de nuestras libertades [acto 3 ]. (Aplausos.) Y diré algo más [subacto 1], señorías (subacto 2] [acto 3]. Todos los que se sienten demócratas deberían tener un objetivo esencial: [subacto 3] poner toda la energía en la defensa de la vida, de la libertad, de la seguridad, de los concejales del Partido Popular y del Partido Socialista, de los concejales del Partido Socialista y del Partido Popular y de todos los ciudadanos que en Euskadi sufren amenaza y chantaje [subacto 4] [acto 4]. Ese es nuestro compromiso esencial [subacto 5] y debería de ser -espero que lo sea definitivamente [acto 6]- el de todos aquellos que son [ subacto 6] y se sienten demócratas ante todo [subacto 7], que es el compromiso de defender la libertad de todos [subacto 8] y de los que se sienten amenazados [subacto 9] [acto 5] [ENUNCIADO 2].

\section{CONSTATACIÓN DEL HECHO O CONSECUENCIAS}

Subtema 3. Cuente ahí el Gobierno, como ya sabe, desde el primer momento [subacto 10], con todo nuestro apoyo para esos fines [subacto 11] [acto 6]. [ENUNCIADO 3]. 


\begin{tabular}{l|c|c}
\hline \multicolumn{2}{c}{ RESUMEN DE UNIDADES ILOCUTIVO-TEXTUALES } \\
Y DE PROCESAMIENTO
\end{tabular}

Zapatero quiere manifestar con firmeza su compromiso con el país, compromiso que desea "reiterar con toda contundencia", pues es "nuestro compromiso firme" asegura en un caso, y "nuestro compromiso esencial", segundos después, adjetivos ambos concluyentes. En auxilio de tales sintagmas, en su estrategia reforzadora, emplea el mecanismo de la serie enumerativa, dicha, como ocurre en la mayoría de sus emisiones, de forma pausada y remarcando prosódicamente determinados elementos. De este modo, añade más convicción a lo emitido, hasta el punto de llegar a despertar los aplausos de sus correligionarios:

(8) va a ser, como siempre, firme defensor de la Constitución, del Estatuto de Autonomía del País Vasco y de las reglas del juego que en ella se contienen

Esta fuerza argumentativa se acrecienta con el marcador de progresión temática y relación lineal articuladora de tipo continuativo "y diré algo más", introductor de un segundo miembro que viene a reforzar el anterior:

(9) Y diré algo más, señorías. Todos los que se sienten demócratas deberían tener un objetivo esencial: poner toda la energía en la defensa de la vida, de la libertad, de la seguridad

de los concejales del Partido Populary del Partido Socialista, 


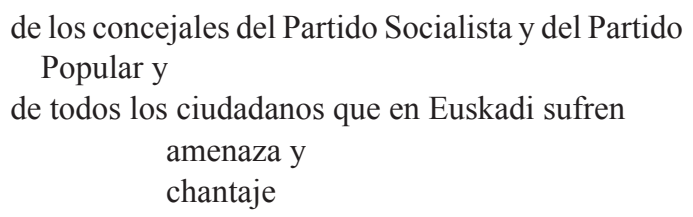

Por tanto, el expresidente socialista utiliza diferentes mecanismos para poner en pie su estrategia, que es enfatizar su argumento; entre ellos, hemos de señalar repeticiones, adjetivos calificativos, el marcador continuativo como introductor de un acto que refuerza lo ya dicho en el previo, o las estructuras enumerativas con elementos comunes iniciales (de la, en el primer nivel de la serie, y de los, en el segundo), lo que añade un tono especial al discurso. Sabemos que la adhesión de un interlocutor a una tesis tiene diferentes grados de intensidad; en el caso del discurso político se suelen potenciar todos los medios para enfatizar la convicción de las propuestas y de ahí la proliferación de mecanismos que auspicien tal objetivo.

No nos ha de extrañar, por tanto, que sean parecidas las herramientas empleadas por Rajoy [2006] cuando afronta un tema de urgencia tan delicado para él y para la mayoría de españoles como es la obligación de mostrar la actitud de su partido en relación con "la voluntad del señor Rodríguez Zapatero de iniciar el diálogo con la banda terrorista ETA". En la citada actitud del líder popular existe, desde el inicio de la intervención, un gran recelo ante la actuación del Gobierno, recelo que se justifica por "las extrañas circunstancias" en que se han llevado a cabo las negociaciones, producto de "las oscilantes convicciones" del ministro del interior; solo tales detalles justifican que el Gobierno haya admitido "las exigencias inaceptables" de ETA. A la contundencia de estos adjetivos, se unen otras formas y mecanismos ya citados previamente; entre ellos, marcadores textuales continuativos como introductores de actos argumentativos reforzadores o las series enumerativas, cuyo funcionamiento vimos previamente.

A las figuras retóricas vistas, se suma en esta intervención la preterición o paralipsis, con la cual Rajoy 'amenaza' al adversario con la mención de cuestiones de las que podría hablar, aunque no lo vaya a hacer. Con ello, por un lado, subraya errores del rival político a la par que prepara su pregunta clave: "lo que me interesa de ese mundo es si están dispuestos o no a dejar las armas", para la que el líder de la derecha sospecha la dificultad de su oponente en la respuesta. Veamos el fragmento:

(10) Señor presidente, señorías, deseo establecer la posición de mi grupo en relación con la voluntad del señor Rodríguez Zapatero de iniciar el diálogo con la banda terrorista ETA. 
- No comentaré, aunque me preocupan, las extrañas circunstancias en que el presidente del Gobierno comunicó a la nación su voluntad de iniciar este diálogo en un acto de su partido en la ciudad de Baracaldo.

- Tampoco quiero entrar en las oscilantes convicciones del señor ministro del Interior quien, sin que sepamos por qué, pasó en menos de cinco días de no estar convencido del alto el fuego a considerarlo completo y real.

- No entraré en nada de esto.

- Tampoco haré ningún comentario sobre las exigencias inaceptables, porque son las de siempre, que surgen del mundo de ETA-Batasuna.

- Lo que me interesa de ese mundo es si están dispuestos o no a dejar las armas.

No se trata de nada de eso, pero todo queda dicho.

La disquisición se presenta con una estructura en forma de serie enumerativa de cuatro elementos, dos de ellos iniciados por no + futuro y los otros dos, como refuerzos argumentativos de los anteriores, precedidos por el marcador textual continuativo negativo tampoco; es obvio que el interés de Rajoy es que cada uno de los cuatro elementos sea una razón que refuerce el conjunto de su argumentación, esencial a la hora de prestigiar lo que está por decir: la pregunta clave. Se vale de una serie enumerativa de dos niveles (primer nivel bipartito y segundo nivel tripartito) iniciados todos sus elementos con verbos en infinitivo:

(11) Espero que se trate de

conocer sus intenciones y

comprobar si existe una decisión irreversible de

abandonar las armas,

disolver la banda y

pedir perdón a las víctimas

El aplauso de sus incondicionales, que se produce en medio de su inicio, llega precedido en primer lugar de un acto consecutivo reforzador de lo anterior, introducido por de acuerdo con ello, y, especialmente, de otra serie enumerativa, figura que, una vez más, propicia tal actitud:

(12) De acuerdo con ello, el Partido Popular no prestará su apoyo a ninguna clase de negociación que tenga como objeto pagar un precio político bien sea

a ETA,

a sus cómplices o

a sus sostenedores. (Aplausos.—Un señor diputado: ¡Muy bien!) 
Rajoy busca, además, un cierre concluyente y quiere que la consecuencia de lo dicho quede enmarcada con claridad, lo que hace mediante el empleo del marcador de progresión temática y relación jerárquica bidireccional argumentativa con matiz consecutivo: en consecuencia; con él, pretende realzar la parte más importante de este tema de urgencia: lo determinante de su propuesta:

(13) En consecuencia, Batasuna no podrá presentarse a las elecciones (subacto 29] mientras no cumpla los requisitos establecidos en la ley.

En el cuadro $\mathrm{n}^{\circ} 5$ se puede observar tanto dicha disposición como las unidades temático-textuales y de procesamiento en que se divide este tema de urgencia:

\section{Cuadro $n^{0}$ 5. Rajoy [2006]. Disposición interna del tema de urgencia en el inicio}

PRESENTACión Del TEMA

SubTema 1. Señor presidente, señorías [subacto 1], deseo establecer la posición de mi grupo en relación con la voluntad del señor Rodríguez Zapatero de iniciar el diálogo con la banda terrorista ETA [subacto 2]. [ENUNCIADO 1].

\section{RAZONES QUE SOSTIENEN TAL OPINIÓN}

SubTEMA 2. No comentaré, aunque me preocupan [subacto 3], las extrañas circunstancias en que el presidente del Gobierno comunicó a la nación su voluntad de iniciar este diálogo en un acto de su partido en la ciudad de Baracaldo [subacto 4] [acto 1]. Tampoco quiero entrar en las oscilantes convicciones del señor ministro del Interior quien, sin que sepamos por qué [subacto 5], pasó en menos de cinco días de no estar convencido del alto el fuego a considerarlo completo y real [subacto 6] [acto 2]. No entraré en nada de esto [acto 3]. Tampoco haré ningún comentario sobre las exigencias inaceptables [subacto 7], porque son las de siempre, que surgen del mundo de ETA-Batasuna [subacto 8] [acto 4]. Lo que me interesa de ese mundo es si están dispuestos o no a dejar las armas [acto 5] [ENUNCIADO 2]

SubTema 3. Señorías [subacto 9], de acuerdo con lo establecido en el Pacto por las libertades y contra el terrorismo corresponde al Gobierno de España dirigir la lucha antiterrorista para lograr la derrota de los criminales [subacto10], utilizando todos los instrumentos del Estado de derecho [subacto 11] [ acto 5]. [ENUNCIADO 3]. El Gobierno, por datos que dice que obran en su poder [subacto 12], ha dado por bueno el cese de las actividades delictivas de ETA [subacto 13] y considera que ha llegado el momento de ponerse en contacto con los terroristas [subacto 14] [acto 6]. Espero que se trate de conocer sus intenciones 
[subacto 15] y comprobar si existe una decisión irreversible de abandonar las armas [subacto 16], disolver la banda [subacto 17] y pedir perdón a las víctimas [subacto 18] [acto 7]. Si es con este fin [subacto 19], el Partido Popular, como firmante del Pacto por las libertades y en coherencia con el mismo [subacto 20], apoyará al Gobierno [subacto 21] [acto 8] [ENUNCIADo 4]. Debe quedar bien entendido, como señala el citado Pacto por las libertades [subacto 20], que de la violencia terrorista no se extraerá en ningún caso ventaja o rédito político alguno [subacto 21] [acto 9]. De acuerdo con ello, el Partido Popular no prestará su apoyo a ninguna clase de negociación que tenga como objeto pagar un precio político [subacto 22] bien sea a ETA, a sus cómplices o a sus sostenedores [subacto 23] [acto 10]. (Aplausos.-Un señor diputado: ¡Muy bien!). ETA debe perder toda esperanza de lograr ni uno solo de los objetivos por los que ha estado matando [subacto 24], entre otros la autodeterminación y la anexión de Navarra [subacto 25] [acto 11]. Es obvio también que el hecho de que se inicie esta verificación no significa que se suspenda ninguna de las funciones del Estado de derecho [subacto 26], especialmente las que corresponden al fiscal general del Estado y a las Fuerzas y Cuerpos de Seguridad [subacto 27] ni que pierda su vigencia la Ley General de Partidos [subacto 28] [acto 12] [ENUNCIADO 5].

\section{CONSTATACIÓN DEL HECHO O CONSECUENCIAS}

Subtema 4. En consecuencia, Batasuna no podrá presentarse a las elecciones (subacto 29] mientras no cumpla los requisitos establecidos en la ley [subacto 30] [acto 13]. [ENUNCIADO 6].

\begin{tabular}{|c|c|c|}
\hline \multicolumn{3}{|c|}{$\begin{array}{l}\text { RESUMEN DE UNIDADES ILOCUTIVO- } \\
\text { TEXTUALES Y DE PROCESAMIENTO }\end{array}$} \\
\hline Temas & SUB(SUB)TEMAS & ENUNCIADOS Y (SUB) ACTOS \\
\hline $\begin{array}{l}\text { Tema2. } \\
\text { Tema de } \\
\text { urgencia. La } \\
\text { oposición } \\
\text { a n t e e } 1 \\
\text { diálogo del } \\
\text { Gobierno } \\
\text { con ETA }\end{array}$ & $\begin{array}{l}\text { Subtema } 1 \text { Manifestación inicial } \\
\text { Subtema } 2 \text { Concesiones al } \\
\text { gobierno } \\
\text { Subtema } 3 \text { obligaciones del } \\
\text { gobierno con ETA. } \\
\text { Subtema } 4 \text {. Batasuna y las } \\
\text { elecciones. }\end{array}$ & $\begin{array}{l}\text { Enunciado } \mathbf{1 .} \\
\text { Enunciado } \mathbf{2} \text {. } \\
\text { Enunciados } \mathbf{3}, \mathbf{4} \text { y } \mathbf{5} \\
\text { Enunciado } \mathbf{6} .\end{array}$ \\
\hline
\end{tabular}




\section{CONCLUSIONES}

El presente artículo pretende, a partir del análisis de los DEN, un mejor conocimiento de la secuencia inicio, y más concretamente de uno de los temas que se incluye en ella: el tema de urgencia. Este no muestra un tipo de discurso que mantenga o repita ciertas formas o mecanismos; al contrario, son fragmentos heterogéneos tanto en el contenido como en la estructura. Tal heterogeneidad la hemos podido advertir al comparar las intervenciones del presidente y del líder de la oposición ante los mismos hechos: la muerte de turistas españoles en Yemen [2007] o de soldados en Afganistán [2011]. El acercamiento lo hemos llevado a cabo desde dos planos diferentes: el plano secuencial, que nos ha conducido al análisis de componentes temáticos, subtemáticos o subsubtemáticos, así como a la disposición interna de tales temas (presentación, razones y consecuencias), y el plano enunciativo, que nos llevó a conocer qué mecanismos discursivos son los empleados por los líderes políticos con el objeto de reforzar las causas del dolor, indignación, patriotismo, etc.; así, hemos analizado las funciones que cumplen en estos temas de urgencia determinados conectores continuativo-reforzadores, adjetivos, series enumerativas o la preterición.

\section{REFERENCIAS BIBLIOGRÁFICAS}

CoRTÉs, LuIs. 2007. Las series enumerativas en el debate político tras el atentado de Barajas: Rodríguez Zapatero y Rajoy frente a frente. Oralia 10: 47-102.

2012a. Los límites del discurso: condicionantes y realizaciones. CLAC. Círculo de Lingüistica Aplicada a la Comunicación 51: 3-49. Disponible en http://www.ucm.es/ info/circulo/no51/cortes.pdf.

2012b. La serie enumerativa y su función en el cierre de los discursos. I. Los discursos del presidente. Estudios Filológicos 49: 39-57.

En prensa. Sobre inicios y cierres en el discurso político. Estudios Filológicos 51.

(coord.). 2008. La serie enumerativa en el discurso oral en español, Madrid: Arco/Libros.

Cortés, Luis y María Matilde CAmacho. 2005. Unidades de segmentación y marcadores del discurso. Madrid: Arco/Libros.

GARRIDO, JoAQUín. 2011. Unidades y relaciones en la construcción del discurso. En Actas del IX Congreso de Lingüística General, pp. 976-990. Valladolid: Universidad de Valladolid.

Robles Garrote, Pilar. 2013. La conferencia como género monológico: análisis macroestructural en español e italiano. Boletín de Filología XLVIII, 1: 127-146. 